\title{
THE DEPENDENCE OF THE FIRST EIGENVALUE OF THE INFINITY LAPLACIAN WITH RESPECT TO THE DOMAIN
}

\author{
J. C. NAVARRO, J. D. ROSSI, A. SAN ANTOLIN \\ Departamento de Análisis Matemático, Universidad de Alicante, \\ Ap. Correos 99, 03080 Alicante Spain \\ e-mails: \{jc.navarro, julio.rossi, angel.sanantolin\}@ua.es \\ and N. SAINTIER \\ Departamento de Matemática, FCEyN Universidad de Buenos Aires (1428) Buenos Aires, \\ Argentina, and Instituto de Ciencias - Universidad Nacional de General Sarmiento, \\ J. M. Gutierrez 1150, C.P. 1613 Los Polvorines, Pcia de Bs. As., Argentina \\ e-mails:nsaintie@dm.uba.ar;nsaintie@ungs.edu.ar
}

(Received 11 August 2012; revised 6 November 2012; accepted 24 January 2013; first published online 2 September 2013)

\begin{abstract}
In this paper we study the dependence of the first eigenvalue of the infinity Laplace with respect to the domain. We prove that this first eigenvalue is continuous under some weak convergence conditions which are fulfilled when a sequence of domains converges in Hausdorff distance. Moreover, it is Lipschitz continuous but not differentiable when we consider deformations obtained via a vector field. Our results are illustrated with simple examples.
\end{abstract}

2010 Mathematics Subject Classification. 35J60, 35P30.

1. Introduction. Eigenvalue problems have been studied by many authors and is by now a classical subject. Maximization or minimization of eigenvalues is an active subject of research (see the survey [9]). Let $\Omega \subset \mathbb{R}^{N}$ be a bounded domain, and consider $\Delta_{p} u=\operatorname{div}\left(|\nabla u|^{p-2} \nabla u\right)$, the $p$-Laplacian. Associated with the $p$-Laplacian, there is a sequence of eigenvalues (note that, in general, it is not known if this sequence constitutes the whole spectrum), that is, a sequence $\lambda_{k}$ such that there are nontrivial solutions to the problem

$$
\begin{cases}-\Delta_{p} u=\lambda_{k}|u|^{p-2} u, & \Omega, \\ u=0, & \partial \Omega,\end{cases}
$$

see $[7, \mathbf{1 5}]$. It is also known that the first eigenvalue is isolated, simple (see $[\mathbf{1}, \mathbf{1 4}])$ and can be variationally characterized as

$$
\lambda_{1, p}(\Omega)=\inf _{u \in W_{0}^{1, p}(\Omega)} \frac{\|\nabla u\|_{L^{p}(\Omega)}^{p}}{\|u\|_{L^{p}(\Omega)}^{p}} .
$$

In [8] the dependence of the first eigenvalue with respect to the domain is studied. There it is proved that when the domain is perturbed following a vector field (see below), the 
derivative of $\lambda_{1, p}(\Omega)$ is given by

$$
D_{V} \lambda_{1, p}(\Omega)=-(p-1) \int_{\partial \Omega}\langle V, \eta\rangle\left|\frac{\partial \phi_{1}}{\partial \eta}\right|^{p} d \sigma .
$$

Here $V$ is the deformation field, $\eta$ is the exterior unit normal to $\partial \Omega$ and $\phi_{1}$ is the associated positive eigenfunction normalized according to $\left\|\phi_{1}\right\|_{L^{p}(\Omega)}=1$.

The limit operator $\lim _{p \rightarrow \infty} \Delta_{p}=\Delta_{\infty}$ is the $\infty$-Laplacian given by $\Delta_{\infty} u=$ $\left\langle D^{2} u D u, D u\right\rangle=\sum_{i, j=1}^{N} \frac{\partial u}{\partial x_{j}} \frac{\partial^{2} u}{\partial x_{j} \partial x_{i}} \frac{\partial u}{\partial x_{i}}$ in the sense that solutions to $\Delta_{p} u_{p}=0$ with the Dirichlet data $u_{p}=f$ on $\partial \Omega$ converge as $p \rightarrow \infty$ to the solution to $\Delta_{\infty} u=0$ with $u=f$ on $\partial \Omega$ in the viscosity sense (see $[\mathbf{3}, \mathbf{5}, \mathbf{6}])$. This operator appears naturally when one considers absolutely minimizing Lipschitz extensions in $\Omega$ of a boundary data $f$ (see $[2,3,11])$.

The limit as $p \rightarrow \infty$ of the eigenvalue problem was studied in $[\mathbf{1 2}, \mathbf{1 3}]$, and an anisotropic version in studied in [4]. In these papers the authors prove that

$$
\lim _{p \rightarrow \infty}\left[\lambda_{1, p}(\Omega)\right]^{1 / p}=\lambda_{1}(\Omega)=\inf _{v \in W_{0}^{1, \infty}(\Omega)} \frac{\|\nabla v\|_{L^{\infty}(\Omega)}}{\|v\|_{L^{\infty}(\Omega)}} .
$$

In addition, we have existence of extremals, i.e. functions where the above infimum is attained. These extremals can be constructed taking the limit as $p \rightarrow \infty$ in the eigenfunctions of the $p$-Laplacian eigenvalue problem (see [13]) and are viscosity solutions of the following eigenvalue problem (called the infinity eigenvalue problem in the literature):

$$
\begin{cases}\min \left\{|D u|-\lambda_{1} u,-\Delta_{\infty} u\right\}=0 & \text { in } \Omega, \\ u=0 & \text { on } \partial \Omega .\end{cases}
$$

Note that it is not known if this first eigenvalue is simple.

By looking at the variational quotient that defines $\lambda_{1}(\Omega)$, in [13] it is also proved that the first eigenvalue has a simple geometrical characterization, and it holds that

$$
\lambda_{1}(\Omega)=\frac{1}{R(\Omega)},
$$

where $R(\Omega)$ is the largest possible radius of a ball contained in $\Omega$. This characterization of the first eigenvalue will be a key tool in our analysis.

Our main goal in this paper is to look at the dependence of this first eigenvalue for the infinity Laplacian, $\lambda_{1}(\Omega)$, with respect to the domain. Note that the equation for the eigenfunctions, (1.3), is not linear, not in divergence form, and, in addition, no regularity result is known for the eigenfunctions (further than continuity and the fact that they belong to $W_{0}^{1, \infty}(\Omega)$ ). Also remark that the variational quotient (1.2) does not involve $L^{p}$-integrals but the $L^{\infty}$-norm that is not differentiable. In addition, even for $\Omega$ being a polygon it is hard to compute $R(\Omega)$. These facts make the study of the dependence of $\lambda_{1}(\Omega)$ on the domain a nontrivial task.

Firstly, we show that $\lambda_{1}(\Omega)$ depends continuously on $\Omega$. To this end we let $\Omega_{t}$ be a bounded sequence of domains (we assume that there exists a compact set $K$ with $\left.\Omega_{t} \subset K, \forall t\right)$ that converge to $\Omega$ as $t \rightarrow 0$ in the following sense:

Given $B \subset \subset \Omega$, it holds that $B \subset \subset \Omega_{t}, \forall t$ small enough. 
If $A \subset \subset \Omega_{t_{j}}$ for a subsequence $t_{j} \rightarrow 0$, then $A \subset \subset \Omega$.

Let us denote by $\lambda_{1}\left(\Omega_{t}\right)$ the first eigenvalue of the infinity Laplacian in $\Omega_{t}$.

THEOREM A. The eigenvalue $\lambda_{1}(\Omega)$ is continuous with respect to $\Omega$. More precisely, let $\Omega_{t}$ be a sequence converging to $\Omega$ in the sense that both (1.5) and (1.6) are verified, then

$$
\lim _{t \rightarrow 0} \lambda_{1}\left(\Omega_{t}\right)=\lambda_{1}(\Omega)
$$

Remark that if we have the Haussdorf distance (see [16]) between $\Omega$ and $\Omega_{t}$,

$$
\mathbb{H}\left(\Omega_{t}, \Omega\right):=\max \left\{\sup _{x \in \Omega_{t}} \inf _{y \in \Omega}|x-y| ; \sup _{x \in \Omega} \inf _{y \in \Omega_{t}}|x-y|\right\},
$$

goes to zero then (1.5) and (1.6) are fulfilled. Therefore, we have

$$
\lim _{t \rightarrow 0} \mathbb{H}\left(\Omega_{t}, \Omega\right)=0 \Rightarrow \lim _{t \rightarrow 0} \lambda_{1}\left(\Omega_{t}\right)=\lambda_{1}(\Omega) .
$$

The convergence given by (1.5) and (1.6) is weaker than the convergence in the Hausdorf distance as the following example shows. In dimension two, let $\Omega=] 0,1\left[^{2}\right.$ and $\left.\left.\Omega_{t}=\Omega \cup(]-1 / t, 0\right] \times\right] t+t^{2}, t+t^{3}[)$. Then (1.5) and (1.6) hold but $\mathbb{H}\left(\Omega_{t}, \Omega\right) \geq$ $1 / t \rightarrow \infty$ as $t \rightarrow 0$.

Next, we study the first variation with respect to a vector field (also called shape derivative). To this end, we consider a continuous vector field $V: \mathbb{R}^{N} \rightarrow \mathbb{R}^{N}$, the deformation field and for small $t \in \mathbb{R}$, the perturbed domains

$$
\Omega_{t}=(I d+t V)(\Omega)=\{x+t V(x), x \in \Omega\},
$$

see [10] and [17] for details concerning these kinds of deformations. Thus, our aim is to study the behaviour of $\lambda_{1}\left(\Omega_{t}\right)-\lambda_{1}(\Omega)$ as $t \rightarrow 0$. We find that the first eigenvalue is Lipschitz continuous but, in general, not differentiable. This fact has to be contrasted with the previously mentioned differentiability of the first eigenvalue of the $p$-Laplacian.

THEOREM B. Consider deformations of the domain given by (1.7), then the first eigenvalue $\lambda_{1}\left(\Omega_{t}\right)$ is Lipschitz continuous with respect to $t$ at $t=0$. More precisely, there exists a constant $C=C(\Omega, V)$ such that

$$
\left|\lambda_{1}\left(\Omega_{t}\right)-\lambda_{1}(\Omega)\right| \leq C|t|
$$

for every $t$ small enough.

Note that our results imply that the largest radius, $R(\Omega)$, of a ball contained in $\Omega$ depends continuously with respect to the domain, and, in addition, it is Lipschitz continuous. However, $\lambda_{1}\left(\Omega_{t}\right)$ may not be differentiable with respect to $t$ at $t=0$ when one considers deformations of the domain driven by a vector field. Indeed, there exists a domain $\Omega$ and a vector field $V$ such that

$$
\lim _{t \rightarrow 0+} \frac{\lambda_{1}\left(\Omega_{t}\right)-\lambda_{1}(\Omega)}{t} \neq \lim _{t \rightarrow 0-} \frac{\lambda_{1}\left(\Omega_{t}\right)-\lambda_{1}(\Omega)}{t} .
$$

We include an example that shows this lack of differentiability in Section 3. 
However, there are explicit examples for which $\lambda_{1}\left(\Omega_{t}\right)$ is differentiable at $t=0$ and, moreover, in some cases the derivative can be explicitly computed. We collect these examples in the final section.

2. Continuous dependence. Our aim in this section is to prove Theorem A, our general continuity result of $\lambda_{1}(\Omega)$ with respect to $\Omega$. Note that here we are not restricting ourselves to deformations given by a vector field.

Proof of Theorem $A$. We use the characterization of $\lambda_{1}(\Omega)$ given by (1.4), therefore our task is to show continuity of $R(\Omega)$, the largest radius of a ball contained in $\Omega$.

Let $x_{0} \in \Omega$ be a point such that there is a ball included in $\Omega$ centred at that point with the largest possible radius such that

$$
B\left(x_{0}, R(\Omega)\right) \subset \Omega .
$$

Then, for every $\varepsilon>0$, we have

$$
B\left(x_{0}, R(\Omega)-\varepsilon\right) \subset \subset \Omega .
$$

By (1.5) we also have

$$
B\left(x_{0}, R(\Omega)-\varepsilon\right) \subset \subset \Omega_{t},
$$

and we conclude that, for $t$ small enough, it holds

$$
R\left(\Omega_{t}\right) \geq R(\Omega)-\varepsilon .
$$

As $\varepsilon$ is arbitrary, we conclude that

$$
\liminf _{t \rightarrow 0} R\left(\Omega_{t}\right) \geq R(\Omega) .
$$

Now let

$$
B\left(x_{t}, R\left(\Omega_{t}\right)\right) \subset \Omega_{t} .
$$

Since all $\Omega_{t}$ are contained in a fixed compact set $K$ for every $t$, we can extract a subsequence $t_{j} \rightarrow 0$ such that

$$
x_{j} \rightarrow x_{0} \quad \text { and } \quad R\left(\Omega_{j}\right) \rightarrow R_{0} .
$$

Then for a fixed $\varepsilon>0$ we have for $t$ small enough

$$
B\left(x_{0}, R_{0}-\varepsilon\right) \subset \subset B\left(x_{t}, R\left(\Omega_{t}\right)\right) \subset \Omega_{t},
$$

and hence passing to the limit and using (1.6) we get

$$
B\left(x_{0}, R_{0}-\varepsilon\right) \subset \Omega \text {. }
$$

Hence, as this holds for any $\varepsilon>0$, we get that any possible limit of $R\left(\Omega_{t}\right), R_{0}$, verifies

$$
R_{0} \leq R(\Omega)
$$


and we conclude that

$$
\limsup _{t \rightarrow 0} R\left(\Omega_{t}\right) \leq R(\Omega)
$$

This ends the proof.

3. Lipschitz dependence. In this section we prove Theorem B. As we mentioned in the Introduction, we restrict ourselves to deformations of the form

$$
\Omega_{t}=(I d+t V)(\Omega)=\{x+t V(x), x \in \Omega\}
$$

and study the quotients

$$
\frac{\lambda_{1}\left(\Omega_{t}\right)-\lambda_{1}(\Omega)}{t}
$$

Proof of Theorem B. Again we use the characterization of $\lambda_{1}(\Omega)$ in terms of the largest radius of a ball included in $\Omega$ that is given by (1.4). We have

$$
\begin{aligned}
R\left(\Omega_{t}\right)^{2}= & \max _{x_{t} \in \Omega_{t}} \min _{y_{t} \in \partial \Omega_{t}}\left|x_{t}-y_{t}\right|^{2} \\
= & \max _{x \in \Omega} \min _{y \in \partial \Omega}|x-y+t(V(x)-V(y))|^{2} \\
= & \max _{x \in \Omega} \min _{y \in \partial \Omega}|x-y|^{2} \\
& +2 t\langle(x-y),(V(x)-V(y))\rangle+t^{2}|V(x)-V(y)|^{2} .
\end{aligned}
$$

Therefore, there exists a constant $C$ depending on $\Omega$ and $V$ such that for $t$ small,

$$
\left|R\left(\Omega_{t}\right)^{2}-R(\Omega)^{2}\right| \leq C|t|,
$$

and then the Lipschitz continuity of $R(\Omega)$ follows using that $R(\Omega)$ is continuous (by our previous result, Theorem A). In fact,

$$
\frac{\left|R\left(\Omega_{t}\right)-R(\Omega)\right|}{|t|}=\frac{\left|R\left(\Omega_{t}\right)^{2}-R(\Omega)^{2}\right|}{\left[R\left(\Omega_{t}\right)+R(\Omega)\right]|t|} \leq \frac{C}{R(\Omega)}
$$

for every $t$ small enough.

Next, we present a simple example that shows that $R(\Omega)$ (and hence $\lambda_{1}(\Omega)$ ) is not differentiable. Let us consider $\Omega=B(0,1)$ in $\mathbb{R}^{2}$ and the vector field $V(x, y)=(0, y)$. Then

$$
R(\Omega)=1
$$

and

$$
R\left(\Omega_{t}\right)= \begin{cases}1 & t \geq 0 \\ 1+t & t<0\end{cases}
$$

Therefore, we get

$$
\lim _{t \rightarrow 0+} \frac{R\left(\Omega_{t}\right)-R(\Omega)}{t}=0 \neq 1=\lim _{t \rightarrow 0-} \frac{R\left(\Omega_{t}\right)-R(\Omega)}{t},
$$

and conclude that $R(\Omega)$ is not differentiable. 
4. Examples. In this section we collect simple examples that illustrate the behaviour of $\lambda_{1}(\Omega)$.

EXAMPLE 1. Firstly, let us compute the derivative when the deformation given by the vector field $V$ does not affect the boundary of $\Omega$ near the points where the closure of the maximal balls touch the boundary. In this case the derivative of $\lambda_{1}(\Omega)$ is zero.

Given a domain $\Omega$, let us define the set of contact points as the set of points on the boundary that are touched by the closure of a ball of radius $R(\Omega)$ included in $\Omega$, that is,

$$
C P(\Omega)=\left\{x \in \partial \Omega \cap \bar{B}\left(x_{0}, R(\Omega)\right) \text {, for some } B\left(x_{0}, R(\Omega)\right) \subset \Omega\right\}
$$

Note that the number of points in the contact set is always greater or equal than 2 (a ball of the largest possible radius can not touch the boundary of the domain at only one point).

One can show that if the vector field verifies

$$
V \equiv 0
$$

in a neighbourhood of $C P(\Omega)$ then the derivative of $R(\Omega)$, as well as the derivative of $\lambda_{1}(\Omega)$, exists and is zero, that is,

$$
\lim _{t \rightarrow 0} \frac{R\left(\Omega_{t}\right)-R(\Omega)}{t}=\lim _{t \rightarrow 0} \frac{\lambda_{1}\left(\Omega_{t}\right)-\lambda_{1}(\Omega)}{t}=0 .
$$

In fact, it holds that

$$
R\left(\Omega_{t}\right)=R(\Omega)
$$

for every $t$ small enough. To see this we argue as follows: Firstly, from the fact that $V \equiv 0$ near the contact points, we get that

$$
R\left(\Omega_{t}\right) \geq R(\Omega)
$$

for small $t$. Now if $R\left(\Omega_{t}\right)>R(\Omega)$ for a sequence $t \rightarrow 0$, then we have that there are balls $B\left(x_{t}, R\left(\Omega_{t}\right)\right) \subset \Omega_{t}$. Extracting a subsequence, if necessary, we get that $x_{t} \rightarrow x_{0} \in \Omega$ and we already know, from our continuity result, that $R\left(\Omega_{t}\right) \rightarrow R(\Omega)$. Therefore, it is easy to check that the ball $B\left(x_{0}, R(\Omega)\right)$ is contained in $\Omega$ and hence its boundary must intersect $\partial \Omega$ in $C P(\Omega)$ but this fact leads to a contradiction with $V \equiv 0$ near the contact set $C P(\Omega)$.

Note that (4.1) says that the first eigenvalue is not strictly monotone with respect to the domain, in contrast to what happens for the $p$-Laplacian.

Also remark that we have shown that the limit (along subsequences) of maximal balls in $\Omega_{t}, B\left(x_{t}, R\left(\Omega_{t}\right)\right) \subset \Omega_{t}$, is a maximal ball in $\Omega$. Hence, any limit point of a sequence $y_{t}$ belonging to the contact sets $C P\left(\Omega_{t}\right)$ is contained in the contact set of the limit domain $C P(\Omega)$.

There are trivial examples, like balls of the form $B(0,1+t)$, for which the limit of the contact sets $C P(B(0,1+t))$ is exactly the contact set of the limit domain $C P(B(0,1))$. However, if we consider the rectangle $\Omega=] 0,2[\times] 0,1[$ and the vector field 
$V(x, y)=(0, x)$, we obtain that $C P(\Omega)$ is the union of two points and two segments,

$$
\begin{gathered}
C P(\Omega)=\{(0,1 / 2),(2,1 / 2)\} \cup\{(0, x): 1 / 2 \leq x \leq 3 / 2\} \\
\cup\{(1, x): 1 / 2 \leq x \leq 3 / 2\},
\end{gathered}
$$

while the contact set of $\Omega_{t}$ just consists of three points and its limit as $t \rightarrow 0$ is only $\{(3 / 2,0),(3 / 2,1),(2,1 / 2)\}$ that is strictly contained in $C P(\Omega)$.

EXAMPLE 2. Now we present an example in which $R(\Omega)$ is differentiable and the derivative is not zero. Take the simple example of the square $\Omega=]-1,1\left[{ }^{2} \subset \mathbb{R}^{2}\right.$ with the vector field $V$ such that

$$
V(x, y)= \begin{cases}(a, 0) & x=1,-1<y<1 \\ (-b, 0) & x=-1,-1<y<1 \\ (0, c) & -1<x<1, y=1 \\ (0,-d) & -1<x<1, y=-1\end{cases}
$$

we have $R(\Omega)=1$ and

$$
R\left(\Omega_{t}\right)= \begin{cases}1+\min \left\{\frac{a+b}{2} ; \frac{c+d}{2}\right\} t & \text { for } t>0 \\ 1+\max \left\{\frac{a+b}{2} ; \frac{c+d}{2}\right\} t & \text { for } t<0\end{cases}
$$

Therefore, in this case we have that $R(\Omega)$ is differentiable if and only if $a+b=c+d$ and we get that the derivative is given by

$$
\lim _{t \rightarrow 0} \frac{R\left(\Omega_{t}\right)-R(\Omega)}{t}=\frac{a+b}{2} .
$$

Note that in this example we only have to take into account the value of the vector field in a small neighbourhood of the contact points between the largest ball in $\Omega$ (that in this case is $B(0,1))$ and the boundary of $\Omega$. These contact points are given by $(1,0)$, $(0,1),(-1,0)$ and $(0,-1)$. Therefore, we can modify the vector field $V$ away from these points to obtain the same conclusion for a smooth vector field.

Moreover, by the same reason, we can round the corners of the square to obtain an example of a smooth domain and a smooth vector field $V$ for which $\lambda_{1}$ is not differentiable.

EXAMPLE 3. Let us now show how to compute the derivative of $R\left(\Omega_{t}\right)$ at $t=0$ when the domain $\Omega$ is a triangle in $\mathbb{R}^{2}$ with different vertices $a, b, c$ and the considered differentiable vector field, $V$, gives us that $\Omega_{t}$ is also a triangle. Given $t$, the vertices of the triangle $\Omega_{t}\left(\Omega_{0}=\Omega\right)$ will be denoted by

$$
a^{t}=a+t V(a), \quad b^{t}=b+t V(b) \quad \text { and } \quad c^{t}=c+t V(c) .
$$

Moreover, if we write $L_{1}^{t}, L_{2}^{t}, L_{3}^{t}$, we mean the length of the side of the triangle $\Omega_{t}$ joining $a^{t}$ with $b^{t}, b^{t}$ with $c^{t}$ and $c^{t}$ with $a^{t}$ respectively, i.e.

$$
\begin{aligned}
& L_{1}^{t}=\|a+t V(a)-b-t V(b)\|_{\mathbb{R}^{2}}, \\
& L_{2}^{t}=\|b+t V(b)-c-t V(c)\|_{\mathbb{R}^{2}}, \\
& L_{3}^{t}=\|c+t V(c)-a-t V(a)\|_{\mathbb{R}^{2}} .
\end{aligned}
$$



Thus,

Note that $R\left(\Omega_{t}\right)$ is the radius of the incircle or inscribed circle in the triangle $\Omega_{t}$.

$$
R\left(\Omega_{t}\right)=2 \frac{A\left(\Omega_{t}\right)}{L_{1}^{t}+L_{2}^{t}+L_{3}^{t}},
$$

where $A\left(\Omega_{t}\right)$ is the area of $\Omega_{t}$. Further, by the Heron Formula for the area of a triangle, we have

$$
A\left(\Omega_{t}\right)=\sqrt{P^{t}\left(P^{t}-L_{1}^{t}\right)\left(P^{t}-L_{2}^{t}\right)\left(P^{t}-L_{3}^{t}\right)}
$$

where $P^{t}=\frac{1}{2}\left(L_{1}^{t}+L_{2}^{t}+L_{3}^{t}\right)$.

Writing (4.3) in (4.2) we obtain an explicit formula for $R\left(\Omega_{t}\right)$ depending only on $L_{1}^{t}, L_{2}^{t}$ and $L_{3}^{t}$.

Now we observe that $L_{1}^{t}, L_{2}^{t}$ and $L_{3}^{t}$ are differentiable with respect to $t$. Indeed, we have

$$
\begin{aligned}
& \lim _{t \rightarrow 0} \frac{L_{1}^{t}-L_{1}^{0}}{t}=\frac{1}{L_{1}^{0}}\langle V(a)-V(b), a-b\rangle ; \\
& \lim _{t \rightarrow 0} \frac{L_{2}^{t}-L_{2}^{0}}{t}=\frac{1}{L_{2}^{0}}\langle V(b)-V(c), b-c\rangle ; \\
& \lim _{t \rightarrow 0} \frac{L_{3}^{t}-L_{3}^{0}}{t}=\frac{1}{L_{3}^{0}}\langle V(c)-V(a), c-a\rangle .
\end{aligned}
$$

So we have that $R\left(\Omega_{t}\right)$ is differentiable at $t=0$ and its derivative at the origin can be computed explicitly and depends only on the derivative of $L_{j}^{t}, j=1,2,3$.

AcKnOwLedgements. Partially supported by MEC MTM2010-18128 (Spain).

\section{REFERENCES}

1. A. Anane, Simplicité et isolation de la premiere valeur propre du $p$-Laplacien avec poinds, C. R. Acad. Sci. Paris Série I 305, (1987), 725-728.

2. G. Aronsson, Extensions of functions satisfying Lipschitz conditions, Ark. Math. 6 (1967), 551-561.

3. G. Aronsson, M. G. Crandall and P. Juutinen, A tour of the theory of absolutely minimizing functions, Bull. Amer. Math. Soc. 41 (2004), 439-505.

4. M. Belloni and B. Kawohl, The pseudo- $p$-Laplace eigenvalue problem and viscosity solutions as $p \rightarrow \infty$, ESAIM Control Optim. Calc. Var. 10 (2004), 28-52.

5. T. Bhattacharya, E. Di Benedetto and J. Manfredi, Limits as $p \rightarrow \infty$ of $\Delta_{p} u_{p}=f$ and related extremal problems, Rend. Sem. Mat. Univ. Politec. Torino Special issue (1991), 15-68.

6. M. G. Crandall, H. Ishii and P. L. Lions, User's guide to viscosity solutions of secondorder partial differential equations, Bull. Amer. Math. Soc. 27 (1992), 1-67.

7. J. García-Azorero and I. Peral, Existence and non-uniqueness for the $p$-Laplacian: Nonlinear eigenvalues, Comm. Partial Differ. Equ. 12 (1987), 1389-1430.

8. J. Garcia-Melian and J. Sabina de Lis, On the perturbation of eigenvalues for the $p$ Laplacian, C. R. Acad. Sci. Paris Séries I 332 (2001), 893-898.

9. A. Henrot, Minimization problems for eigenvalues of the Laplacian, J. Evol. Equ. 3 (2003), 443-461.

10. A. Henrot and M. Pierre, Variation et optimization de forme, Mathématiques et Applications 48 (Springer, Berlin, Germany, 2005). 
11. R. Jensen, Uniqueness of Lipschitz extensions: Minimizing the sup norm of the gradient, Arch. Rational Mech. Anal. 123 (1993), 51-74.

12. P. Juutinen and P. Lindqvist, On the higher eigenvalues for the $\infty$-eigenvalue problem, Calc. Var. Partial Differ. Equ. 23(2) (2005), 169-192.

13. P. Juutinen, P. Lindqvist and J. J. Manfredi, The $\infty$-eigenvalue problem, Arch. Rational Mech. Anal. 148 (1999), 89-105.

14. P. Lindqvist, On the equation $\operatorname{div}\left(|\nabla u|^{p-2} \nabla u\right)+\lambda|u|^{p-2} u=0$, Proc. Amer. Math. Soc. 109 (1990), 157-164. (Addendum to: On the equation $\operatorname{div}\left(|\nabla u|^{p-2} \nabla u\right)+\lambda|u|^{p-2} u=0$, Proc. Amer. Math. Soc. 116 (1992), 583-584).

15. P. Lindqvist, A nonlinear eigenvalue problem. Topics in Mathematical Analysis, 175203, Ser. Anal. Appl. Comput., 3 (World Scientific, Hackensack, NJ, 2008).

16. J. Munkres, Topology, 2nd ed (Prentice Hall, Upper Saddle River, NJ, 1999).

17. J. Simon, Optimal design for Neumann condition and for related boundary value conditions, in Boundary control and boundary variations, Lecture Notes in Control and Information Sciences, 100 (J. P. Zolezio, Editor) (Springer, New York, 1988). 